\title{
Semicomplete Multipartite Digraphs Whose Every Arc Is Contained in a Hamiltonian Path
}

\author{
Wei Meng* and Shengjia Li \\ School of Mathematical Sciences, Shanxi University, \\ 030006 Taiyuan, P.R. China \\ \{alfred.hofmann, ursula. barth, ingrid. beyer, christine guenther, \\ frank.holzwarth, anna.kramer, erika.siebert-cole, lncs $\}$ @springer.com \\ http://www.sxu.edu.cn
}

\begin{abstract}
A semicomplete multipartite digraph is obtained by replacing each edge of a complete multipartite graph by an arc or by a pair of two mutually opposite arcs. In 2002, L.Volkmann 5] raised a problem: determine some sufficient conditions for semicomplete multipartite digraphs such that every arc is contained in a Hamiltonian path. In this paper, we give a new sufficient condition and describe some semicomplete multipartite digraphs demonstrating that this sufficient condition is independent and in some sense, it is best possible.
\end{abstract}

Keywords: Semicomplete multipartite digraph, Hamiltonian paths, Semi-partition complete digraphs.

\section{Terminology and Introduction}

In this paper all digraphs are finite without loops and multiple arcs. The vertex set and the arc set of a digraph are denoted by $V(D)$ and $A(D)$, respectively. The number of vertices in $D$ is called the order of $D$. A digraph obtained by replacing each edge of a complete $n$-partite graph by an arc or a pair of mutually opposite arcs is called a semicomplete n-partite or multipartite digraph. Obviously, a tournament is a semicomplete multipartite digraph without cycles of length 2 where each partite set contains exactly one vertex.

If $x y$ is an arc of a digraph $D$, then we say that $x$ dominates $y$ and write $x \rightarrow y$. More generally, for disjoint subsets $A$ and $B$ of $V(D)$ or subdigraphs of $D$, if every vertex of $A$ dominates every vertex of $B$, then we say that $A$ dominates $B$, denoted by $A \rightarrow B$. In the case when there is no arc from $B$ to $A$, we write $A \Rightarrow B$. If both of $A \rightarrow B$ and $A \Rightarrow B$ hold, then we say that $A$ strictly dominates $B$, denoted by $A \mapsto B$. Furthermore, if for all $a \in A$ and

\footnotetext{
* Research partially supported by Youth Science Foundation of Shanxi University. This research was conducted while the author was visiting Prof. Hubertus Th.Jongen in Lehrstuhl C für Mathematik of RWTH Aachen. Corresponding author.
} 
$b \in B$, either $a, b$ are not adjacent or $a \rightarrow b$, then we denote this relationship between $A$ and $B$ by $A \hookrightarrow B$. For $S \subseteq V(D)$ and for any vertex $x \in V(D)$, we define $d^{+}(x, S)\left(d^{-}(x, S)\right)$ to be the number of out-neighbours (in-neighbours) of $x$ in $S$. If $S=V(D)$, we also write $d^{+}(x, D)=d^{+}(x)\left(d^{-}(x, D)=d^{-}(x)\right)$. Furthermore, $N^{+}(S)\left(N^{-}(S)\right)$ is the set of out-neighbours (in-neighbours) of $S$.

By a cycle (path) we mean a directed cycle (directed path). A path $P=$ $x_{1} x_{2} \ldots \ldots x_{k}$ starts with the arc $x_{1} x_{2}$, or $x_{1} x_{2}$ is the initial arc of $P$. A cycle of length $m$ is an $m$-cycle. A cycle in a digraph $D$ is Hamiltonian if it includes all the vertices of $D$. A digraph $D$ is Hamiltonian if it contains a Hamiltonian cycle. If $x$ is a vertex on a cycle, then $x^{+}\left(x^{-}\right)$denotes the successor (predecessor) of $x$ on $C$. If a cycle (path) $Q$ contains a subpath from $x$ to $y$, then we denote it by $Q[x, y]$. A subdigraph $F$ of a digraph $D$ which contains all the vertices of $D$ and which consists of a set of vertex-disjoint cycles is called a 1-regular factor. $F$ is said to be minimal if there is no 1-regular factor $F^{\prime}$ of $D$ consisting of less cycles than $F$.

For a vertex set $X$ of digraph $D$, we define $D[X]$ as the subdigraph induced by $X$. A set $X \subseteq V(D)$ is independent if the induced subdigraph $D[X]$ has no arcs. The independent number $\alpha(D)=\alpha$ is the maximum size among the independent sets of vertices of $D$. A digraph $D$ is strong connected or strong if, for each pair of vertices $u$ and $v$, there is a path in $D$ from $u$ to $v$. A digraph $D$ with at least $k+1$ vertices is $k$-connected if for any set $A$ of at most $k-1$ vertices, the subdigraph $D-A$ obtained by deleting $A$ is strong. The connectivity of $D$, denoted by $\kappa(D)$, is then defined to be the largest value of $k$ such that $D$ is $k$-connected.

Some first observation on semicomplete multipartite digraphs can be found in Moon's book [3]. In the following decades graph theorists began to study multipartite semicomplete digraphs more extensively. Excellent sources for more information in this area are found in recent book by Bang-Jensen and Gutin 1 . In 2002, L.Volkmann [5] discussed the longest paths through an arc in strong semicomplete multipartite digraphs and gave a sufficient condition such that all arcs are contained in a Hamiltonian path.

Theorem 1 [5]. Let $D$ be a semicomplete multipartite digraphs with $\kappa(D)>$ $\alpha(D)$, then every arc is contained in a Hamiltonian path.

In addition, L.Volkmann [5] posed the following problem:

Problem 1 [5]. Determine some sufficient conditions for semicomplete multipartite digraphs such that every arc is contained in a Hamiltonian path.

In this paper, we give a new sufficient condition in term of this problem. Furthermore, through examples we illustrate the independence of this sufficient condition and the best possibility in some sense. Moreover, a new class of semicomplete multipartite digraphs are introduced and we present a sufficient condition for these digraphs to be Hamiltonian. 


\section{Preliminaries}

The following results play an important role in our investigation. First the definition of semi-partition complete digraphs which was first introduced by Guo [2] and some properties of these digraphs are given.

Definition 1. Let $D$ be a semicomplete multipartite digraph with the partite sets $V_{1}, V_{2}, \ldots, V_{k}$. If $d^{+}\left(x_{i}, V_{j}\right), d^{-}\left(x_{i}, V_{j}\right) \geq\left|V_{j}\right| / 2$ for every vertex $x_{i} \in V_{i}$ and for every $1 \leq i, j \leq k, j \neq i$, then $D$ is called a semi-partition complete digraph.

Theorem 2 [2]. Let $D$ be a semi-partition complete digraph of order $n$ with $\alpha(D) \leq n / 2$. Then $D$ is Hamiltonian or there exists two vertex-disjoint cycles $C_{1}$ and $C_{2}$ in $D$ such that $V\left(C_{1}\right) \cup V\left(C_{2}\right)=V(D)$ and $\left|V\left(C_{1}\right) \cap V_{j}\right|=\left|V\left(C_{2}\right) \cap V_{j}\right|=$ $\left|V_{j}\right| / 2$ for all $1 \leq j \leq k$, where $V_{1}, V_{2}, \ldots, V_{k}$ are the partite sets of $D$.

Now we state a shorten version of Yeo's [6] result about the structure of semicomplete multipartite digraphs with a 1-regular factor.

Theorem $3[6]$. Let $D$ be a semicomplete multipartite digraph containing a 1-regular factor. Then there exists a 1-regular factor $F$ in $D$ whose cycles can be labeled, $C_{1}, C_{2}, \ldots, C_{t}$, such that the following holds:

There exists a partite set $V^{*}$ in $D$ such that whenever $x_{j} \rightarrow x_{1}$ where $x_{j} \in$ $V\left(C_{j}\right), x_{1} \in V\left(C_{1}\right)$ and $1<j \leq t$, we have $\left\{x_{j}^{+}, x_{1}^{-}\right\} \subseteq V^{*}$.

In particular, if $F$ is minimal, then it has the properties described above.

\section{Main Results}

Theorem 4. Let $D$ be a semicomplete multipartite digraph with partite sets $V_{1}, V_{2}, \ldots, V_{k}$. If $d^{+}\left(x_{i}, V_{j}\right), d^{-}\left(x_{i}, V_{j}\right) \geq\left(\left|V_{j}\right|+1\right) / 2$ for every vertex $x_{i} \in V_{i}$, for each $1 \leq i, j \leq k, j \neq i$, and $\alpha(D) \leq(n-1) / 2$, then every arc is contained in a Hamiltonian path.

Proof. Let $x y$ be any arc of $D$. From the assumption we see that $D-x$ is a semi-partition complete digraph which satisfies $\alpha(D-x) \leq \alpha(D) \leq(n-1) / 2=$ $|V(D-x)| / 2$. According to Theorem $2, D-x$ is either Hamiltonian or there exists two vertex-disjoint cycles $C_{1}$ and $C_{2}$ in $D-x$ such that $V\left(C_{1}\right) \cup V\left(C_{2}\right)=V(D-x)$ and $C_{i}$ contain exactly half of the vertices of every partite set of $D-x$, for $i=1,2$.

If $D-x$ has a Hamiltonian cycle, denoted by $y y_{2} y_{3} \ldots y_{n-1} y$, then $x y$ lies on such a Hamiltonian path of $D x y y_{2} y_{3} \ldots y_{n-1}$. Then we are done.

Now, we assume that there exists two vertex-disjoint cycles $C_{1}$ and $C_{2}$ in $D-x$ satisfying the condition described as above. Assume without loss of generality that $y \in C_{1} \cap V_{1}$. Since $x$ and $y^{-}$are both adjacent to $y, x$ and $y^{-}$do not belong to $V_{1}$. Note that $C_{2} \cap V_{1} \neq \emptyset$, we claim that there exists at least one vertex $z \in C_{2} \cap V_{1}$ such that $y^{-} \rightarrow z$. In fact, if this is not true, then we have $C_{2} \cap V_{1} \mapsto y^{-}$. Hence, $N^{+}\left(y^{-}, V_{1}\right) \subseteq C_{1} \cap V_{1}$ holds. Recalling $x$ does not belong to $V_{1}$, we have $\left|C_{1} \cap V_{1}\right|=\left|V_{1}\right| / 2$. This contradicts the assumption 
$d^{+}\left(y^{-}, V_{1}\right) \geq\left(\left|V_{1}\right|+1\right) / 2$. Now, the arc $x y$ is on such a Hamiltonian path $x y C_{1}\left[y^{+}, y^{-}\right] z C_{2}\left[z^{+}, z^{-}\right]$. This completes the proof.

An example will show that conditions in Theorem 4 are independent to Theorem 1.

Example 1. Let $V_{1}=\left\{v_{1}, v_{2}, v_{3}\right\}, V_{2}=\left\{v_{4}, v_{5}, v_{6}\right\}$, and $V_{3}=\left\{v_{7}\right\}$ be the partite sets of a semicomplete 3 -partite digraphs $D$. The arcs between $v_{1}, v_{2}, v_{3}, v_{4}$ are $v_{1} \rightarrow v_{4} \rightarrow v_{2} \rightarrow v_{4} \rightarrow v_{3}$, and the other arcs between two vertices of different partite sets are two mutually opposite arcs. It is not difficult to check that this digraph $D$ satisfies all conditions in Theorem 4 and hence, every arc is contained in a Hamiltonian path.

Note that the vertices $\left\{v_{5}, v_{6}, v_{7}\right\}$ is a separating set of $D$. So $\kappa(D) \leq 2$, but $\alpha(D)=3$. Therefore, $D$ does not satisfy the conditions in Theorem 1 .

In addition, at the proof of Theorem 4, we actually showed that every arc is not only on a Hamiltonian path, but also the arc is exactly the initial arc of the Hamiltonian path. In this sense, the degree-condition in Theorem 4 is best possible. See the following example.

Example 2. Let $V_{1}=\left\{x_{1}, x_{2}, x_{3}, x_{4}\right\}, V_{2}=\left\{y_{1}, y_{2}, y_{3}, y_{4}\right\}$ and $V_{3}=\left\{z_{1}, z_{2}, z_{3}, z_{4}\right\}$ be the partite sets of a semicomplete 3 -partite digraphs $D$. The arcs between any two vertices in $S_{1}=\left\{x_{1}, y_{1}, z_{1}, x_{2}, y_{2}, z_{2}\right\}$, which belong to different partite sets are defined by two mutually opposite arcs. Similarly, we define the arcs in $S_{2}=\left\{x_{3}, y_{3}, z_{3}, x_{4}, y_{4}, z_{4}\right\}$. Moreover, $S_{1} \hookrightarrow S_{2}$.

It is easy to see that $D$ is a semicomplete 3-partite digraph with order 12, $\alpha(D)=4$ and $d^{+}\left(x_{3}, V_{2}\right)=2$. But every $\operatorname{arc}$ in $S_{2}$ is not on any Hamiltonian path which starts with this arc.

In the following we abstract the conditions in Theorem 4 and define a new class of semicomplete multipartite digraphs. Furthermore, we will present a sufficient condition for these digraphs to be Hamiltonian.

Definition 2. Let $D$ be a semicomplete multipartite digraph with partite sets $V_{1}, V_{2}, \ldots, V_{k}$. If $d^{+}\left(x_{i}, V_{j}\right), d^{-}\left(x_{i}, V_{j}\right) \geq\left(\left|V_{j}\right|+1\right) / 2$ for every vertex $x_{i} \in V_{i}$ and for every $1 \leq i, j \leq k, j \neq i$, then $D$ is called a quasi-semi-partition complete digraph.

Remark 1. According to Definition 2, Theorem 4 can be stated as: Let $D$ be a quasi-semi-partition complete digraph with order $n$ and $\alpha(D)<n / 2$, then every arc of $D$ is contained in a Hamiltonian path.

Remark 2. Clearly, quasi-semi-partition complete digraphs are also semi-partition complete digraphs.

Theorem 5. Every quasi-semi-partition complete digraph is strong. 
Proof. Let $D$ be a quasi-semi-partition complete digraph with partite sets $V_{1}, V_{2}, \ldots, V_{k}$, and $x, y$ any two distinct vertices of $D$.

If $x, y \in V_{i}$ for some $i \in\{1,2, \ldots, k\}$, then for any $j \neq i$, and $1 \leq j \leq k$, let $A_{j}=\left\{a \in V_{j} \mid x \mapsto a\right\}, B_{j}=\left\{b \in V_{j} \mid x \rightarrow b, b \rightarrow x\right\}$, and $C_{j}=\left\{c \in V_{j} \mid c \mapsto x\right\}$. Obviously, $A_{j}, B_{j}, C_{j}$ are pairwise disjoint and $A_{j} \cup B_{j} \cup C_{j}=V_{j}$. Because of $d^{+}\left(x, V_{j}\right) \geq\left(\left|V_{j}\right|+1\right) / 2$, we know $\left|A_{j} \cup B_{j}\right| \geq\left(\left|V_{j}\right|+1\right) / 2$ and then, $\left|C_{j}\right|<\left|V_{j}\right| / 2$. Also note that $d^{-}\left(y, V_{j}\right) \geq\left(\left|V_{j}\right|+1\right) / 2$, so there is at least one vertex in $A_{j} \cup B_{j}$ which dominates $y$, say $u$. According to the definitions of $A_{j}$ and $B_{j}$, xuy is a path in $D$ from $x$ to $y$. Similarly, we can show that there is also a path from $y$ to $x$.

Now we suppose that $x \in V_{i}, y \in V_{j}$ for some $i, j \in\{1,2, \ldots, k\}$ and $i \neq j$. Since $x$ and $y$ are adjacent, we assume without loss of generality that $x \rightarrow y$. If $y \rightarrow x$ also holds, then there is nothing to prove. Otherwise, we have $x \mapsto y$. Let $A=\left\{a \in V_{i} \mid a \mapsto y\right\}, B=\left\{b \in V_{i} \mid y \rightarrow b, b \rightarrow y\right\}, C=\left\{c \in V_{i} \mid y \mapsto c\right\}$, $L=\left\{d \in V_{j} \mid x \mapsto d\right\}, M=\left\{e \in V_{j} \mid x \rightarrow e, e \rightarrow x\right\}$ and $N=\left\{f \in V_{j} \mid f \mapsto x\right\}$. Clearly, $L, M, N$ are pairwise disjoint, $L \cup M \cup N=V_{j}$ and $B \neq \emptyset$. Because of $d^{-}\left(x, V_{j}\right) \geq\left(\left|V_{j}\right|+1\right) / 2$, we know $|M \cup N| \geq\left(\left|V_{j}\right|+1\right) / 2$ and then, $|L|<\left|V_{j}\right| / 2$. Now we claim that there is at least one $\operatorname{arc} u v$ in $D$ from $B$ to $M \cup N$. In fact, if this is not true, then $M \cup N \mapsto B$ and for any vertex $b$ of $B$, we have $N^{+}\left(b, V_{j}\right) \subseteq L$. So $d^{+}\left(b, V_{j}\right) \leq|L|<\left|V_{j}\right| / 2$, a contradiction ! By the definitions of $B, M$ and $N$, we see that $y \rightarrow u$ and $v \rightarrow x$ hold. Hence, yuvx is just a path in $D$ from $y$ to $x$.

In summary, $D$ is strongly connected.

By using Theorem 5, we obtain a sufficient condition for a quasi-semi-partition complete digraph to be Hamiltonian.

Theorem 6. Let $D$ be a quasi-semi-partition complete digraph with $\alpha(D) \leq$ $n / 2$. Then $D$ is Hamiltonian.

Proof. From Remark 2 and Theorem 2, $D$ is either Hamiltonian or there exists two vertex-disjoint cycles $C_{1}$ and $C_{2}$ in $D$ such that $V\left(C_{1}\right) \cup V\left(C_{2}\right)=V(D)$ and $\left|V\left(C_{1}\right) \cap V_{j}\right|=\left|V\left(C_{2}\right) \cap V_{j}\right|=\left|V_{j}\right| / 2$ for all $1 \leq j \leq k$, where $V_{1}, V_{2}, \ldots, V_{k}$ are the partite sets of $D$. We only need to consider the latter case. Obviously, $C_{1}, C_{2}$ is the minimal 1-regular factor. Thus, according to Theorem 3, we may assume that $C_{1}, C_{2}$ and $V^{*}$ just satisfies Theorem 3 . Theorem 5 ensue that $D$ is strong, hence there are vertices $u \in C_{1}$ and $v \in C_{2}$ such that $v \rightarrow u$. Furthermore, $u^{-} \in V^{*}$ and $u^{-} \mapsto V\left(C_{2}\right) \cap\left(V-V^{*}\right)$. In fact, $u^{-}$is adjacent to any vertex of $V\left(C_{2}\right) \cap\left(V-V^{*}\right)$. If there exists a vertex in $V\left(C_{2}\right) \cap\left(V-V^{*}\right)$ which dominates $u^{-}$, then again by Theorem 3 , we deduce $u^{--} \in V^{*}$, but this is clearly not impossible. For any partite set $V^{\prime}$ different from $V^{*}$, we have $d^{-}\left(u^{-}, V^{\prime}\right) \leq\left|C_{1} \cap V^{\prime}\right|=\left|V^{\prime}\right| / 2$. This contradicts the definition of quasi-semi-partition complete digraphs. Therefore, $D$ is Hamiltonian.

Acknowledgements. We would like to express our gratitude to Professor Yubao Guo for some detailed comments and valuable suggestions. 


\section{References}

1. Bang-Jensen,J., Gutin,G.: Digraphs: Theory, Algorithms and Applications. Springer, London (2000)

2. Guo,Y., Tewes,M., Volkmann,L., Yeo,A.: Sufficient conditions for semicomplete multipartite digraphs to be Hamiltonian. Discrete Math. 212 (2000) 91-100

3. Moon,J.W.: Topics on Tournaments. Holt, Rinehart and Winston, New York (1968)

4. Ore,O.: Theory of graphs. Amer. Soc. Colloq. Publ., Vol. 38. (1962)

5. Volkmann,L.: Longest paths through an arc in strong semicomplete multipartite digraphs. Discrete Math. 258 (2002) 331-337

6. Yeo,A.: One-diregular subgraphs in semicomplete multipartite digraphs. J. Graph Theory 24 (1997) 175-185 